\title{
GÉNOMIQUE FÉLINE, PROGRÈS RÉCENTS ET INTÉRÊTS EN RECHERCHE ET EN MÉDECINE VÉTÉRINAIRE
}

\author{
FELINE GENOMICS, PROGRESSES AND INTERESTS IN RESEARCH AND \\ VETERINARY MEDICINE
}

Marie $\mathrm{ABITBOL}{ }^{1}$

Communication présentée le 25 février 2021, manuscrit accepté le 25 avril 2021

\begin{abstract}
RÉSUMÉ
Le séquençage et l'annotation du génome du chat domestique (Felis catus), initialement publiés en 2007, étaient de médiocre qualité. Ils ont depuis été amendés puis complétés par le développement d'outils de génomique qui, combinés à des stratégies génétiques en constante évolution, permettent de rechercher gènes et variants d'intérêt avec efficacité. Ces progrès bénéficient à la médecine vétérinaire féline, mais font également du chat domestique un modèle pour l'étude de la domestication, l'évolution des espèces, mais aussi un modèle biomédical en constante ascension. Progressivement, le chat qui a déjà conquis le cœur des foyers français et du web mondial, est en train de conquérir le cœur des généticiens.
\end{abstract}

Mots-clés : Chat, ADN, Génome, Maladie héréditaire, Modèle animal

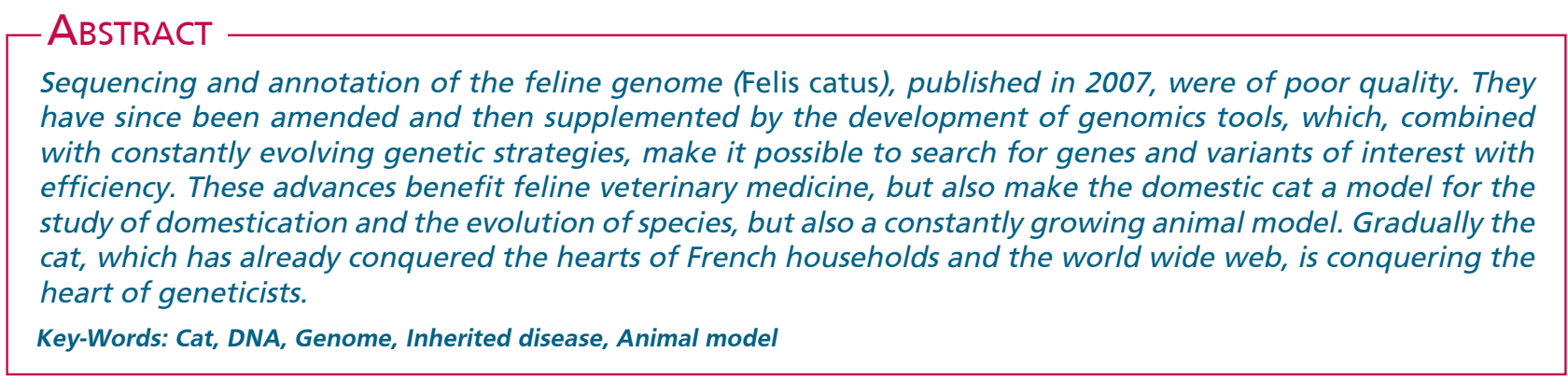

\section{INTRODUCTION}

Le chat (Felis catus) est depuis longtemps apprécié pour sa compagnie. Son succès ne se dément pas puisqu'en dix ans sa population a augmenté de plus de trois millions d'individus en France, passant de 10,7 millions en 2008 à 14,2 millions en 2018 (enquête FACCO/KANTAR TNS 2018, www.facco.fr). Les chats de maison ou "de gouttière" (sans pedigree) sont majoritaires mais les chats de races connaissent un engouement récent comme en témoigne le nombre d'enregistrements de pedigrees au Livre Officiel des Origines Féline (LOOF) qui a plus que doublé en dix ans, en passant de 25099 en 2010 à 52 395 en 2020 (www.loof.asso.fr). En parallèle, la médicalisation du chat a progressé et de nombreuses cliniques vétérinaires spécialement dédiées au chat ont vu le jour. Cet engouement pour le chat domestique s'est accompagné, dans le domaine de la recherche et de la médecine vétérinaire, d'un intérêt croissant pour l'espèce et du développement d'outils d'analyse du génome de Felis catus qui ont considérablement amélioré les possibilités d'analyses génomiques, l'étude de la domestication du chat et l'identification de mutations délétères ou d'intérêt.

(1) Pr Marie Abitbol, VetAgro Sup, Campus vétérinaire de Lyon, 1 avenue Bourgelat 69280 Marcy l'Etoile, et Institut NeuroMyoGène (INMG) 8 avenue Rockefeller 69008 Lyon. Courriel : marie.abitbol@vetagro-sup.fr 


\section{LES PROGRÈS DE LA GÉNOMIQUE FÉLINE}

\section{Les outils de la génétique féline avant l'ère de la génomique}

Les premières études de cytogénétique féline menées dans les années 70 ont montré que le caryotype du chat domestique était constitué de 38 chromosomes répartis en 18 paires d'autosomes et une paire de chromosomes sexuels (Wurster-Hill \& Gray, 1973). Depuis les années 80, des efforts ont été faits pour développer la cartographie du génome félin. Ont notamment été développés : des panels d'hybrides somatiques, puis d'hybrides d'irradiation ; des cartes de liaison, dont certaines ont exploité des croisements entre chats domestiques (Felis catus) et chats léopards du Bengale (Prionailurus bengalensis); des cartes physiques puis les séquençages "basse" puis "haute" couvertures de génomes entiers.

Dans les années 80 et 90, les panels d'hybrides somatiques, créés in vitro grâce à la fusion de cellules de chats et de rongeurs, ont permis de localiser à l'échelle d'un chromosome les premiers loci félins (O'Brien \& Nash, 1982), dont le gène de la tyrosinase, impliqué dans le patron de couleur colourpoint, emblématique du Siamois (O'Brien et al. 1986).

Les panels d'hybrides d'irradiation, également créés en fusionnant des cellules de rongeurs avec des cellules de chats, mais préalablement irradiées (Page \& Murphy, 2008), ont permis de réaliser des cartes à haute résolution sur lesquelles les généticiens ont pu positionner des marqueurs génétiques de type microsatellites mais aussi des loci non polymorphes tels que des gènes. En 1999, une trentaine de loci félins étaient cartographies (Murphy et al. 1999), ouvrant la voie à des cartes d'hybrides d'irradiation de plus en plus denses et résolutives (Murphy et al. 2000 ; Menotti-Raymond et al. 2003a ; Murphy et al. 2007 ; Davis et al. 2009), pour aboutir, en 2012, à la publication d'une carte possédant une résolution d'un marqueur tous les 40 kilobases (kb) (Bach et al. 2012).

En parallèle, l'exploitation de croisements expérimentaux, dont des croisements interspécifiques, a permis de positionner sur une carte de liaison gènes et marqueurs microsatellites, autorisant ainsi la cartographie puis l'identification de mutations impliquées dans des maladies héréditaires et des caractères d'intérêt (Gandolfi \& Alhaddad, 2015). En 1999, la première carte de liaison a été publiée. Elle comportait 253 loci (Menotti-Raymond et al. 1999). Elle sera complétée et combinée aux données des hybrides d'irradiation (Sun et al. 2001 ; Menotti-Raymond et al. 2003b), puis aux données d'un séquençage basse résolution, pour aboutir en 2009 à une carte génétique intégrée et comparée avec l'homme et le chien (Menotti-Raymond et al. 2009a). Finalement, en 2016, une carte de liaison haute résolution a été générée à partir de plus de 450 chats et a permis de positionner plus de 58000 marqueurs de type SNP (Single Nucleotide Polymorphism) sur les chromosomes félins (Li et al. 2016).

En 2007, la première version de la séquence complète du génome d'un chat domestique a été publiée. Il s'agissait d'un séquençage basse résolution (profondeur du séquençage : 1,9 X) du génome d'un chat Abyssin appelé Cinnamon (Pontius et al. 2007). L'assemblage et l'annotation de la séquence de ce génome étaient également peu précis (O'Brien et al. 2008 ; Pontius \& O'Brien, 2009). Cependant, ce premier effort de séquençage a ouvert la voie à la génomique féline.

\section{Les différentes vies du génome félin}

La première version du génome félin, publiée en 2007 (Pontius et al. 2007), a depuis été largement amendée et complétée (pour revue, voir : Zhang \& Schoenebeck, 2020). En effet, cette première version réalisée à partir du séquençage Sanger de clones plasmidiques et fosmidiques (stratégie du shotgun hiérarchique) ne comportait que $50 \%$ du génome couvert par des contigs (assemblages chevauchants de clones contigus) d'une taille de plus de $2,7 \mathrm{~kb}$. Cette valeur, appelée contig N50, permet en particulier d'apprécier la qualité du séquençage et de l'assemblage en termes de fragmentation. Le contig N50 du premier génome félin était donc de 2,7 kb. L'incorporation de données générées par le séquençage des extrémités de clones de type BAC (chromosomes artificiels de bactéries) puis par un re-séquençage de nouvelle génération (dit NGS : Next Generation Sequencing) avec une profondeur de $12 \mathrm{X}$, ainsi que de données issues d'un chat Russe sans pedigree, d'un chat sauvage Européen (Felis silvestris silvestris) et d'une annotation du génome initial, a conduit aux versions appelées Felis_catus_5.0 puis Felis_catus_6.2 du génome félin (www.ensembl.org, Tamazian et al. 2014). Ces différentes versions ont toutes été générées à partir des séquences d'un unique individu de race Abyssin, le chat Cinnamon. Une amélioration notable du génome félin a été publiée en 2014, avec l'incorporation de données issues du séquençage NGS d'un panel de chats de différentes races et de félins sauvages. La profondeur de séquençage est ainsi passée à $20 \mathrm{X}$ et des données concernant le polymorphisme ont été ajoutées (Mullikin et al. 2010). Cependant, cette nouvelle version du génome, appelée Felis_catus_8.0, était toujours fragmentée et comportait des lacunes (plus de 300 000), des inversions, des segments mal assignés ou non assignés à un chromosome (www.ensembl.org) et un contig N50 qui était passé à 2,7 à $45 \mathrm{~kb}$. La dernière amélioration du génome félin date de 2017 et a été publiée fin 2020 (Buckley et al. 2020a). Cette version actuelle, appelée Felis_catus_9.0, intègre des données de séquence issues de séquenceurs de troisième génération permettant de générer des lectures longues [séquences de $12 \mathrm{~kb}$ en moyenne, contre 150 à 900 paires de bases (pb) auparavant]. Les erreurs d'assignation ont été réduites et le génome a considérablement gagné en continuité. Le contig N50 a bondi à près de 42 mégabases $(\mathrm{Mb})$ et la profondeur du séquençage a atteint $72 \mathrm{X}$. Enfin, en décembre dernier, les génomes quasi complets (sans lacune au niveau séquence) d'un chat domestique et d'un chat léopard du Bengale, produits à l'aide des dernières générations de séquenceurs, sont venus compléter le panel des données disponibles pour l'étude du génome félin (Bredemeyer et al. 2020). 


\section{Des puces pour les chats}

Le séquençage du génome de Cinnamon, puis le séquençage basse profondeur $(3 \mathrm{X})$ de six chats de différentes races (American shorthair, Cornish rex, Burmese, Persan, Siamois, Ragdoll) et d'un félin sauvage (Felis silvestris cafra), a permis de découvrir trois millions de marqueurs SNP (Mullikin et al. 2010). Cette ressource a été utilisée pour développer, en 2010, une première puce féline de génotypage. Cette première puce à marqueurs SNP a été commercialisée par la société Illumina (www.illumina.com). Elle est toujours disponible à ce jour. Elle comporte 62 897 marqueurs SNP et a été appelée 63K Illumina Infinium iSelect ${ }^{\circledR}$ DNA array. Avec la publication du génome de Cinnamon, la commercialisation de cette puce a très largement contribué à l'essor de la génétique et de la génomique félines. En effet, jusqu'en 2010, la cartographie de loci morbides ou d'intérêt passait par l'utilisation de marqueurs de type microsatellites, dont l'usage est plus long, plus coûteux et moins résolutif que les marqueurs SNP d'une puce. Les études pangénomiques étaient donc rares et l'essentiel des études était donc de type gène-candidat (voir partie 3).

Avec un peu plus de 60000 marqueurs, la puce Illumina féline est une puce basse densité. Elle permet de cartographier des loci mendéliens mais elle n'est pas assez résolutive pour cartographier des QTL (Quantitative Trait Locus). De plus, les trois versions successives du génome de référence du chat domestique n'ont pas facilité l'utilisation de cette puce, dont les coordonnées des marqueurs avaient initialement été assignées sur Felis_catus_6.2. La réassignation des SNP sur Felis_catus_8.0 a été réalisée en 2018 (Gandolfi et al. 2018). La dernière réassignation sur Felis_catus_9.0 a été publiée fin 2020 (Buckley et al. 2020a).

A la suite de la puce commercialisé pour l'espèce canine (Axiom $^{\mathrm{TM}}$ Canine HD Array, www.thermofisher.com), une deuxième génération de puce à marqueurs SNP est en cours de développement chez le chat. Cette nouvelle puce, haute densité, qui sera commercialisée par la société ThermoFisher, devrait comporter 650000 marqueurs (source personnelle). Cette puce haute densité devrait permettre à l'espèce féline de rattraper son retard sur l'espèce canine et ainsi autoriser l'étude de caractères héréditaires complexes (Gandolfi \& Alhaddad, 2015 ; Gandolfi et al. 2018 ; Cook et al. 2020).

\section{Le projet 99 Lives}

Aux États-Unis, le professeur Leslie Lyons de l'Université du Missouri est à l'origine d'un projet et d'un consortium de chercheurs dédié au séquençage de génomes félins, intitulé 99 Lives (http://felinegenetics.missouri.edu/). Le projet vise à créer et exploiter une ressource de génomique féline constituée de génomes entiers de chats domestiques de différentes races, sans pedigree et de félins sauvages. Les données de séquence sont accessibles aux membres du consortium mais également à toute la communauté scientifique, une première série de 195 génomes ayant été publiée (Buckley et al. 2020b). L'analyse de ces 195 génomes a permis de générer un fichier de variants génomiques félins qui aide, par comparaison, à l'identification de nouveaux variants délétères ou d'intérêt (Katz et al. 2020 ; Yu et al. 2020).

\section{DES PROGRÈS AU SERVICE DE L'AMÉLIORA- TION DES CONNAISSANCES SUR LE CHAT DOMESTIQUE}

\section{Origine des chats modernes, domestication et hybridation}

Les premières traces de domestication du chat dateraient de près de 9500 ans (Vigne et al. 2004). Les premières études moléculaires, réalisées à l'aide de marqueurs nucléaires de type microsatellites et de séquences mitochondriales, ont montré que les chats domestiques modernes dériveraient essentiellement de chats sauvages du Proche Orient, de l'espèce Felis silvestris lybica (Driscoll et al. 2007). Ces données n'ont pas été remises en cause par les analyses menées à l'aide d'outils de génomique récents (NGS, puces à SNP) mais il est apparu que les données générées avec des marqueurs microsatellites et l'analyse de l'ADN mitochondrial ne permettaient pas de résoudre tous les questionnements autour de la domestication et de l'origine de certaines populations animales (Hunter, 2018).

Des études complémentaires sont en cours, à l'aide du séquençage NGS de génomes entiers de chats domestiques anciens et modernes et de chats sauvages, afin d'élucider les relations complexes que les chats ont entretenu avec l'homme au cours du processus de domestication, des migrations de populations humaines et animales et les évènements d'hybridation successifs entre chats domestiques et chats sauvages qui ont conduit aux chats modernes actuels.

Enfin, les marqueurs SNP se sont avérés plus pertinents que les marqueurs microsatellites utilisés auparavant, pour détecter des évènements d'hybridation entre chats domestiques et chats sauvages Européens (Felis silvestris silvestris), évènements d'intérêt dans le contexte de la préservation des espèces sauvages et de la biodiversité (Oliveira et al. 2015 ; Steyer et al. 2018).

\section{Parenté entre races félines}

L'utilisation de la puce $63 \mathrm{~K}$ Illumina Infinium iSelect ${ }^{\circledR}$ a permis de confirmer la parenté entre races de chats et la structure de la population féline. Ainsi en 2018 (Gandolfi et al. 2018), ont été confirmées les données acquises dix ans auparavant, à partir de marqueurs microsatellites et d'un petit nombre de marqueurs SNP (Menotti-Raymond et al. 2008 ; Lipinski et al. 2008). Trois grands clusters de races ont été identifiés : les chats d'Asie, d'Europe de l'ouest et d'Europe centrale (Gandolfi et al. 2018).

\section{DES PROGRÈS AU SERVICE DE LA GÉNÉ- TIQUE MÉDICALE VÉTÉRINAIRE}

Les progrès de la génomique féline ont surtout contribué à l'identification de gènes et de mutations gouvernant des phénotypes délétères ou d'intérêt félins. 


\section{De l'approche gène candidat au clonage positionnel}

Les premières études visant à rechercher des mutations impliquées dans des caractères héréditaires félins ont été conduite à l'aide d'une méthode appelée l'approche gène-candidat. Cette méthode consiste à utiliser les connaissances acquises dans une autre espèce pour faire l'hypothèse qu'un gène puisse être un bon candidat pour expliquer un caractère chez le chat. Par exemple, lorsqu'une maladie héréditaire est caractérisée phénotypiquement chez le chat et que le tableau clinique, anatomopathologique ou histologique est similaire à celui d'une affection caractérisée chez l'homme ou la souris, et pour laquelle une mutation causale a été identifiée, on fait l'hypothèse que le gène félin orthologue du gène humain ou de souris est muté chez le chat. On cherche alors des variants dans la séquence du gène félin candidat, qui pourraient expliquer le tableau clinique. De nombreuses mutations ont été identifiées à l'aide de cette méthode, qui a pour avantages d'être simple et en général peu coûteuse (les coûts dépendent en partie de la taille du gène à séquencer). La première limite de la méthode est que l'on teste la candidature de gènes déjà connus : on ne peut pas identifier de nouveaux gènes. La deuxième limite est que l'on risque de passer à côté d'une mutation causale dans un autre gène que le gène candidat testé si le caractère étudié est hétérogène génétiquement (plusieurs gènes en cause pour un même phénotype). Cependant, citons comme exemples les succès d'identification des mutations de la polykystose rénale du Persan (Lyons et al. 2004), des couleurs chocolat et cannelle (Lyons et al. 2005). Plus récemment ont également été identifiées les mutations du syndrome d'alopécie congénitale du Sacré de Birmanie (Abitbol et al. 2015a), des couleurs ambre, russet et carnelian (Peterschmitt et al. 2009 ; Gustafson et al. 2017 ; Abitbol \& Gache 2019, Figure 1) à l'aide d'approches gène-candidat.
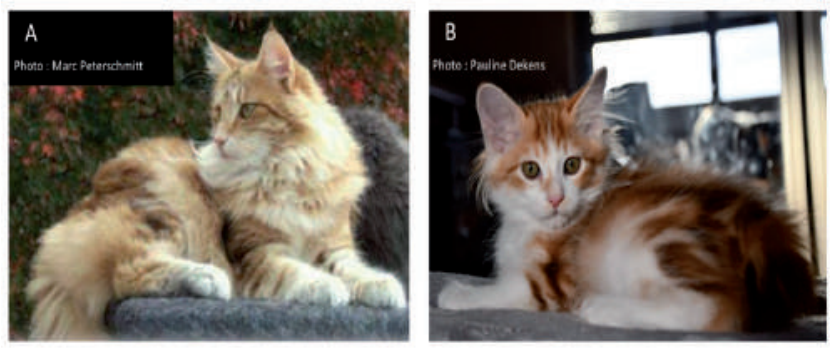

Figure 1 : Phénotypes ambre et carnelian chez le chat. A) Photographie d'un chat Norvégien ambre, homozygote pour l'allèle e du gène MC1R (Melanocortin 1 Receptor). B) Photographie d'un chaton Kurilian bobtail carnelian, homozygote pour l'allèle $e^{c} d u$ gène MC1R. Les allèles e et ec ont été identifiés par l'approche gène candidat.

Le développement de cartes du génome félin et de marqueurs génétiques a permis l'émergence de nouvelles stratégies de recherche de mutations. Les panels de marqueurs microsatellites puis la puce $63 \mathrm{~K}$ Illumina Infinium iSelect ${ }^{\circledR}$ ont autorisé les approches dites de clonage positionnel, qui visent à rechercher une mutation à partir de sa position dans le génome, sans faire d'hypothèse de départ sur le gène en cause. Les analyses de liaison familiales (bien adaptées aux marqueurs microsatellites), qui exploitaient les relations de parentés entre individus, se sont développées les premières ; puis les études d'association et la cartographie d'homozygotie (mieux adaptées au génotypage sur puce à marqueurs SNP, Figure 2). Des analyses de liaison ont par exemple permis de cartographier les loci de la panachure blanche (Cooper et al. 2006) ou du silver (Menotti-Raymond et al. 2009b) et d'identifier la mutation de l'amyotrophie spinale du Maine coon (Fyfe et al. 2006). Le réel essor de la recherche de mutations chez le chat est cependant venu des études utilisant les données de génotypage générées avec la puce Illumina. Il est devenu possible de cartographier un locus à partir d'un faible nombre de sujets. Ainsi, des études d'association ou de cartographie d'homozygotie ont permis de mettre en évidence de nombreuses mutations félines de maladies héréditaires mendéliennes telles que la myasthénie congénitale du Sphynx et du Devon rex (Abitbol et al. 2015b), la myopathie hypokaliémique du Burmese (Gandolfi et al. 2012) ou de caractères phénotypiques comme le poil frisé du Selkirk rex (Gandolfi et al. 2013) et l'albinisme oculocutané du Donskoy (Mériot et al. 2020). Dans le futur, ce même type d'étude d'association permettra sans doute de mettre en évidence d'autres mutations notamment grâce au développement de la deuxième génération de puce à marqueurs SNP à haute densité.

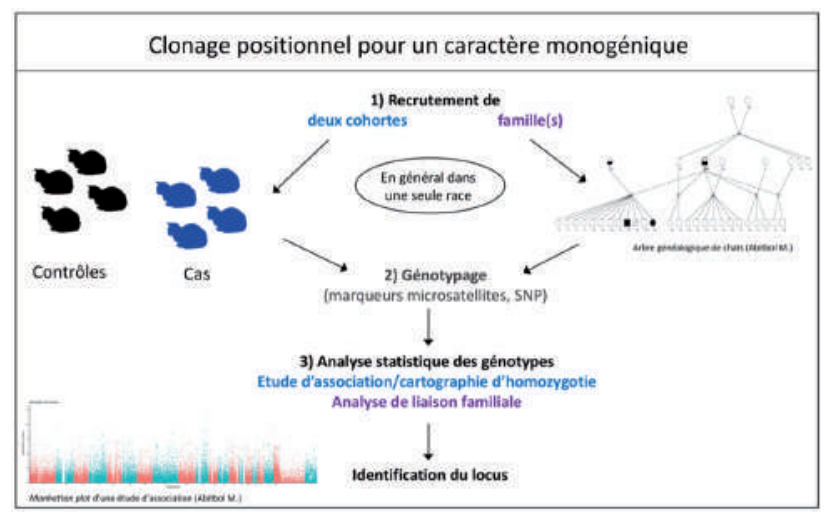

Figure 2 : Stratégies de clonage positionnel. Pour une étude d'association, une cohorte d'individus cas et une cohorte d'individus contrôles sont constituées. Dans le cas d'une analyse familiale de liaison, une ou des familles sont recrutées. Les individus sont génotypés à l'aide de marqueurs microsatellites (préférés dans le cas de l'analyse de liaison) ou SNP (préférés dans le cas des études d'association) et une analyse statistique des génotypes permet de rechercher le locus en cause et de le cartographier.

\section{Du clonage positionnel au séquençage des génomes}

Les plus récentes évolutions qu'ont connues les stratégies d'identification de mutations chez le chat sont venue de l'augmentation de la vitesse et de la baisse du coût du séquençage. Avec les techniques NGS (Next Generation Sequencing) est désormais possible de séquencer un génome de chat complet avec une profondeur de $30 \mathrm{X}$ (profondeur moyenne requise pour rechercher une mutation) en quelques jours et pour moins de 1000 euros. Il n'est plus nécessaire de recruter des cohortes de chats atteints et contrôles, en théorie un seul individu atteint suffit 
pour identifier une mutation. Rechercher une mutation chez un seul individu nécessite de comparer la séquence de cet individu à une référence. Si l'on séquence par exemple le génome d'un chat Persan et que l'on compare sa séquence à la séquence de référence Felis_catus_9.0, des dizaines de milliers de variants génomiques seront mis en évidence, le génome de référence ayant été obtenu à partir d'un chat de race Abyssin. Il est donc nécessaire de pouvoir filtrer les variants qui relèvent du polymorphisme entre races et entre individus. Séquencer un unique individu ne permet donc pas d'identifier une mutation. Il est nécessaire de disposer d'une base de données de génomes félins déjà séquencés, qui serviront à filtrer les variants. Avant la création du projet 99 Lives par le Pr Leslie Lyons, cette base de données n'existait pas. Désormais, la communauté scientifique dispose des génomes de 195 chats de différentes races (Buckley et al. 2020b). Plusieurs mutations félines ont déjà été identifiées grâce au projet 99 Lives (Buckley et al. 2020b ; Li et al. 2020 ; Yu et al. 2020 ; http://felinegenetics.missouri.edu/). Plus cette base de données de génomes félins sera enrichie, plus il sera possible de rechercher des mutations à partir d'une séquence unique.

\section{Dépistage et conseil génétique}

L'identification de gènes et de mutations impliqués dans des maladies héréditaires félines a ouvert la voie à la commercialisation de tests ADN de diagnostic et de dépistage, à l'usage des vétérinaires, des éleveurs et des propriétaires de chats. Ces tests bénéficient à la médecine féline en améliorant le diagnostic des affections héréditaires et en évitant de laisser naître des chatons prédisposés ou atteints de maladies souvent incurables. Des tests $\mathrm{ADN}$ individuels, des packs de tests races-spécifiques, mais aussi des panels de tests "toutes races", sont désormais commercialisés qui générèrent des inquiétudes en termes d'interprétation des résultats et d'usage en sélection. S'il est incontestable que les progrès de la génomique féline bénéficient au bien-être et à la santé des chats, ces progrès soulèvent désormais de nouvelles questions (Lyons 2015 ; Moses et al. 2018 ; Lyons \& Buckley 2020).

Les panels de tests, réalisés à l'heure actuelle à l'aide de puces SNP sur mesure basse densité (quelques milliers de SNP), ou de séquençage ciblé, permettent également de générer des données de génotypage pangénomiques utilisables dans des études d'association. Les laboratoires commercialisant ces panels [MyDogDNA (https://mydogdna.com/) et Embark (https://embarkvet.com/) chez le chien ; MyCatDNA (https://mycatdna.com/) et BasePaws (https://basepaws.com/) chez le chat] peuvent utiliser les données pour rechercher des mutations (Deane-Coe et al. 2018) ou identifier la présence de certains variants dans des populations ou des races où ils n'avaient pas été décrits antérieurement (Donner et al. 2016).

\section{DES PROGRÈS QUI CONDUISENT A LA MÉDE- CINE DE PRÉCISION}

Médecine de précision et médecine personnalisée

Le progrès de la génomique féline, les panels de tests et les possibilités ouvertes par le séquençage de génomes entiers conduisent à envisager, pour l'espèce féline, la possibilité de proposer une médecine personnalisée du même type que celle qui est développée pour l'homme. Tout propriétaire de chat, présentant en consultation un animal avec des signes évocateurs d'une affection héréditaire ou néoplasique, pourrait se voir proposer le séquençage du génome entier, ou du génome des cellules tumorales, de son animal. Il deviendrait alors possible d'adapter le suivi et le traitement proposé en fonction du génotype de l'animal.

Par exemple, des mutations germinales dans le gène MDR1 (Multi Drug Resistance Protein 1 ou ABCB1 : ATP Binding Cassette Subfamily B Member 1), responsable d'une sensibilité médicamenteuse chez le chien et le chat, pourraient être recherchées spécifiquement avant toute utilisation d'une molécule à risque (Mealey, 2016). Des mutations somatiques de KIT (KIT Proto-Oncogene, Receptor Tyrosine Kinase) pourraient être recherchées dans les biopsies tumorales, afin d'adapter l'usage éventuel de l'imatinib $^{\mathrm{TM}}$ (Bonkobara, 2015). En cas de tableau clinique de myasthénie, il peut être pertinent d'écarter toute mutation responsable de myasthénie congénitale féline, notamment dans le gène COLQ (Collagen Like Tail Subunit Of Asymmetric Acetylcholinesterase, Abitbol et al. 2015) avant de proposer un traitement classique à base d'anticholinestérasiques pour de la myasthénie grave. En effet, ces derniers aggravent les symptômes de myasthénie congénitale alors qu'il existe un traitement spécifique pour les individus portant des mutations de COLQ (éphédrine ou salbutamol ; Finsterer, 2019). C'est notamment le cas des chats myasthéniques, des races Sphynx et Devon rex, qui peuvent bénéficier, lorsqu'ils sont mutés COLQ, d'un traitement à base de salbutamol (Figure 3). Ou encore, en cas de coagulopathie, de nombreux gènes pouvant être en cause, une recherche pangénomique de variants, par séquençage, peut être entreprise afin d'adapter le traitement (Li et al. 2020).
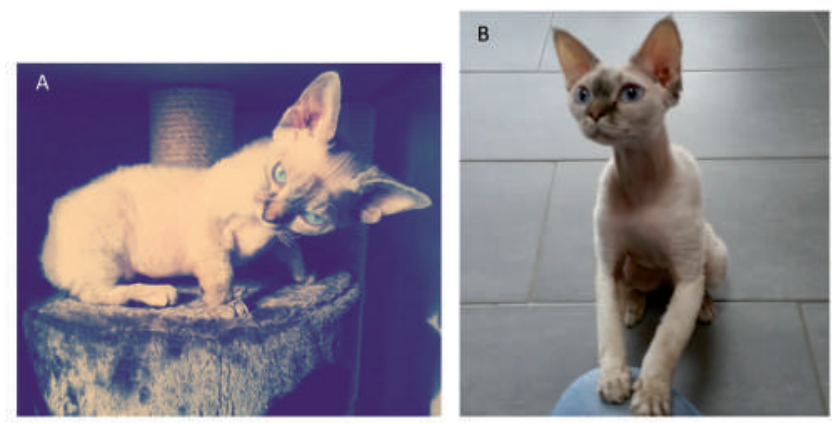

Figure 3 : Médecine personnalisée chez le chat. A) Chatte Devon rex atteinte de myasthénie congénitale, avant traitement. La chatte n'arrive pas à tenir sa tête relevée du fait d'une faiblesse musculaire généralisée. B) Même chatte après trois mois de traitement avec du salbutamol. La chatte arrive à tenir sa tête droite et une fonction musculaire normale a été restaurée (Photos: K. Hubner).

\section{Le chat peut-il faire mieux que la souris ?}

Si l'antériorité, la facilité de manipulation et d'utilisation et la pertinence du modèle souris ne peuvent pas être remises en cause en génétique médicale comparée, ce modèle ne permet pas de résoudre toutes les questions biologiques posées par l'étude des affections génétiques (maladies héréditaires simples et complexes, cancers). Les exemples de publications ne sont pas 
rares où l'invalidation totale ou partielle de la fonction d'un gène chez la souris ne permet pas de modéliser le phénotype humain correspondant. Des modèles animaux spontanés comme le chien se révèlent parfois plus pertinents (Story et al. 2020). En cancérologie, la souris n'est pas toujours le modèle le plus adapté (Hédan et al. 2020 ; van der Weyden et al. 2020 ; Prouteau \& André, 2019 ; Willmann et al. 2019). De plus, les modèles animaux de taille intermédiaire, comme le chien ou le chat, permettent des études précliniques poussées permettant de combler le vide entre le modèle souris et l'homme (Hédan et al. 2020 ; Tarone et al. 2019 ; Nghiem \& Kornegay, 2019 ; Bentley et al. 2017). Si le modèle canin est désormais reconnu (Copeland et al. 2008 ; Lequarré et al. 2011), dernièrement, le modèle félin a prouvé son intérêt en génétique médicale comparée (Lyons, 2020). L'identification d'une mutation dans le gène PEA15 (Proliferation and Apoptosis Adaptor Protein 15), chez des chats atteints d'anomalies du développement cérébral, a montré que le modèle félin était parfois plus pertinent que le modèle souris. En effet, les souris invalidées dans PEA15 ne présentaient pas de phénotype. Le chat a ainsi fourni un nouveau gène candidat pour les lissencéphalies humaines (Graff et al. 2020), comme il avait déjà fourni de nouveaux candidats pour une affection oculaire (Cogné et al. 2020) ou du nanisme (Buckley et al. 2020a). La montée en puissance et les retombées récentes du projet 99 Lives témoignent de l'intérêt du chat en génétique médicale comparée. Enfin, les efforts de mise en banque d'échantillons félins, menés dans différents pays, complètent le panel des ressources disponibles pour les généticiens qui travaille en génétique féline. En France, la biobanque Feli-DNA, membre du réseau CRB-Anim (www.crb-anim.fr), a été développée en collaboration entre l'EnvA, VetAgro Sup et Antagene (www.antagene.com) et financée grâce au Programme d'Investissement d'Avenir (PIA ANR-11-INBS-0003).

\section{CONCLUSION}

Les progrès de la génomique féline sont récents, mais ils ont connu une croissance exponentielle ces dix dernières années. Ils bénéficient à la génétique féline, à la médecine vétérinaire, à l'élevage et à la génétique médicale comparée. Après avoir conquis la planète, nos foyers et le web, le chat pourrait-il détrôner la souris qui habite le cour des généticiens?

\section{REMERCIEMENTS}

L'auteur remercie les vétérinaires référents, les propriétaires et les éleveurs de chats, qui contribuent à la recherche en génétique féline en fournissant prélèvements, informations de santé, pedigrees et photographies.

\section{BIBLIOGRAPHIE}

- Abitbol M, Bossé P, Thomas A, Tiret L. A deletion in FOXN1 is associated with a syndrome characterized by congenital hypotrichosis and short life expectancy in Birman cats. PLoS One. 2015a Mar 17; 10(3): e0120668.

- Abitbol M, Hitte C, Bossé P, Blanchard-Gutton N, Thomas A, Martignat L, et al. COLQ Missense Mutation in Sphynx and Devon Rex Cats with Congenital Myasthenic Syndrome. PLoS One. 2015b Sep 1; 10(9): e0137019.

- Abitbol M, Gache V. Copal, a new MC1R allele in the domestic cat. Anim Genet. 2019 Oct; 50(5): 553-554.

- Bach LH, Gandolfi B, Grahn JC, Millon LV, Kent MS, Narfstrom K, et al. A high-resolution 15,000(Rad) radiation hybrid panel for the domestic cat. Cytogenet Genome Res. 2012; 137(1): 7-14.

- Bentley RT, Ahmed AU, Yanke AB, Cohen-Gadol AA, Dey M. Dogs are man's best friend: in sickness and in health. Neuro Oncol. 2017 Mar 1; 19(3): 312-322.
- Bonkobara M. Dysregulation of tyrosine kinases and use of imatinib in small animal practice. Vet J. 2015 Aug; 205(2): 180-8.

- Bredemeyer KR, Harris AJ, Li G, Zhao L, Foley NM, Roelke-Parker M, et al. Ultracontinuous single haplotype genome assemblies for the domestic cat (Felis catus) and Asian leopard cat (Prionailurus bengalensis). J Hered. 2020 Dec 11: esaa057.

- Buckley RM, Davis BW, Brashear WA, Farias FHG, Kuroki K, Graves T, et al. A new domestic cat genome assembly based on long sequence reads empowers feline genomic medicine and identifies a novel gene for dwarfism. PLoS Genet. 2020a Oct 22; 16(10): e1008926.

- Buckley RM, Gandolfi B, Creighton EK, Pyne CA, Bouhan DM, LeRoy $\mathrm{ML}$, et al. Werewolf, There Wolf: Variants in Hairless Associated with Hypotrichia and Roaning in the Lykoi Cat Breed. Genes (Basel). 2020b Jun 22; 11(6): 682.
- Cogné B, Latypova X, Senaratne LDS, Martin L, Koboldt DC, Kellaris G, et al. Mutations in the Kinesin-2 Motor KIF3B Cause an Autosomal-Dominant Ciliopathy. Am J Hum Genet. 2020 Jun 4; 106(6): 893-904.

- Cook SR, Conzemius MG, McCue ME, Ekenstedt KJ. SNP-based heritability and genetic architecture of cranial cruciate ligament rupture in Labrador Retrievers. Anim Genet. 2020 Oct; 51(5): 824-828.

- Cooper MP, Fretwell N, Bailey SJ, Lyons LA. White spotting in the domestic cat (Felis catus) maps near KIT on feline chromosome B1. Anim Genet. 2006 Apr; 37(2): 163-5.

- Copeland H, Dukes-McEwan J, Sargan D, Kennedy L, Starkey M, Hendricks A, Callanan S. LUPA - studying human diseases using dog genetics. Vet Rec. 2008 Nov 1; 163(18): 550.

- Davis BW, Raudsepp T, Pearks Wilkerson AJ, Agarwala R, Schäffer AA, Houck $\mathrm{M}$, et al. A high-resolution cat radiation hybrid and integrated FISH 
mapping resource for phylogenomic studies across Felidae. Genomics. 2009 Apr; 93(4): 299-304.

- Deane-Coe PE, Chu ET, Slavney A, Boyko AR, Sams AJ. Direct-to-consumer DNA testing of 6,000 dogs reveals 98.6-kb duplication associated with blue eyes and heterochromia in Siberian Huskies. PLoS Genet. 2018 Oct 4; 14(10): e1007648.

- Donner J, Kaukonen M, Anderson H, Möller F, Kyöstilä K, Sankari S, et al. Genetic Panel Screening of Nearly 100 Mutations Reveals New Insights into the Breed Distribution of Risk Variants for Canine Hereditary Disorders. PLoS One. 2016 Aug 15; 11(8): e0161005.

- Finsterer J. Congenital myasthenic syndromes. Orphanet J Rare Dis. 2019 Feb 26; 14(1): 57.

- Fyfe JC, Menotti-Raymond M, David VA, Brichta L, Schäffer AA, Agarwala $\mathrm{R}$, et al. An approximately 140-kb deletion associated with feline spinal muscular atrophy implies an essential LIX1 function for motor neuron survival. Genome Res. 2006; 16(9): 1084-90.

- Gandolfi B, Gruffydd-Jones TJ, Malik R, Cortes A, Jones BR, Helps CR, et al. First WNK4-hypokalemia animal model identified by genome-wide association in Burmese cats. PLoS One. 2012; 7(12): e53173.

- Gandolfi B, Alhaddad H, Joslin SE, Khan R, Filler S, Brem G, Lyons LA. A splice variant in KRT71 is associated with curly coat phenotype of Selkirk Rex cats. Sci Rep. 2013; 3: 2000.

- Gandolfi B \& Alhaddad H. Investigation of inherited diseases in cats: genetic and genomic strategies over three decades. J Feline Med Surg. 2015; 17(5): 405-15.

- Gandolfi B, Alhaddad H, Abdi M, Bach LH, Creighton EK, Davis BW, et al. Applications and efficiencies of the first cat 63K DNA array. Sci Rep. 2018; 8(1): 7024.

- Graff EC, Cochran JN, Kaelin CB, Day K, Gray-Edwards HL, Watanabe $\mathrm{R}$, et al. PEA15 loss of function and defective cerebral development in the domestic cat. PLoS Genet. 2020; 16(12): e1008671.

- Gustafson NA, Gandolfi B, Lyons LA. Not another type of potato: MC1R and the russet coloration of Burmese cats. Anim Genet. 2017; 48(1): 116-120.

- Hédan B, Rault M, Abadie J, Ulvé R, Botherel N, Devauchelle P, et al. PTPN11 mutations in canine and human disseminated histiocytic sarcoma. Int J Cancer. 2020; 147(6): 1657-1665.

- Hunter P. The genetics of domestication: Research into the domestication of livestock and companion animals sheds light both on their "evolution" and human history. EMBO Rep. 2018; 19(2): 201-205.

- Katz ML, Buckley RM, Biegen V, O'Brien DP, Johnson GC, Warren WC, Lyons LA. Neuronal Ceroid Lipofuscinosis in a Domestic Cat Associated with a DNA Sequence Variant That Creates a Premature Stop Codon in CLN6. G3 (Bethesda). 2020; 10(8): 2741-2751.

- Li G, Hillier LW, Grahn RA, Zimin AV, David VA, Menotti-Raymond M, et al. A High-Resolution SNP Array-Based Linkage Map Anchors a New Domestic Cat Draft Genome Assembly and Provides Detailed Patterns of Recombination. G3 (Bethesda). 2016; 6(6): 1607-16.

- Lequarré AS, Andersson L, André C, Fredholm M, Hitte C, Leeb T, et al. LUPA: a European initiative taking advantage of the canine genome architecture for unravelling complex disorders in both human and dogs. Vet J. 2011; 189(2): 155-9.

- Li RHL, Ontiveros E, Nguyen N, Stern JA, Lee E, Hardy BT; 99 Lives Cat Genome Consortium. Precision medicine identifies a pathogenic variant of the ITGA2B gene responsible for Glanzmann's thrombasthenia in a cat. J Vet Intern Med. 2020; 34(6): 2438-2446.

- Lipinski MJ, Froenicke L, Baysac KC, Billings NC, Leutenegger CM, Levy $\mathrm{AM}$, et al. The ascent of cat breeds: genetic evaluations of breeds and worldwide random-bred populations. Genomics. 2008; 91(1): 12-21.

- Lyons LA \& Buckley RM. Direct-to-Consumer Genetic Testing for Domestic Cats. Vet Clin North Am Small Anim Pract. 2020 Sep; 50(5): 991-1000.

- Lyons LA, Biller DS, Erdman CA,
Lipinski MJ, Young AE, Roe BA, et al. Feline polycystic kidney disease mutation identified in PKD1. J Am Soc Nephrol. 2004; 15(10): 2548-55.

- Lyons LA, Foe IT, Rah HC, Grahn RA. Chocolate coated cats: TYRP1 mutations for brown color in domestic cats. Mamm Genome. 2005; 16(5): 356-66.

- Lyons LA. Precision medicine in cats-The right biomedical model may not be the mouse! PLoS Genet. 2020; 16(12): e1009177.

- Lyons LA. DNA mutations of the cat: the good, the bad and the ugly. J Feline Med Surg. 2015 Mar; 17(3): 203-19..

- Mealey K. How Should I Treat Dogs \& Cats with MDR1 Mutation? Plumb's Therapeutics Brief Advertisers. 2016: 12-15.

- Menotti-Raymond M, David VA, Lyons LA, Schäffer AA, Tomlin JF, Hutton MK, O'Brien SJ. A genetic linkage map of microsatellites in the domestic cat (Felis catus). Genomics. 1999; 57(1): 9-23.

- Menotti-Raymond M, David VA, Agarwala R, Schäffer AA, Stephens R, O'Brien SJ, Murphy WJ. Radiation hybrid mapping of 304 novel microsatellites in the domestic cat genome. Cytogenet Genome Res. 2003a; 102(1-4): 272-6.

- Menotti-Raymond M, David VA, Roelke ME, Chen ZQ, Menotti KA, Sun S, et al. Second-generation integrated genetic linkage/radiation hybrid maps of the domestic cat (Felis catus). J Hered. 2003b; 94(1): 95-106.

- Menotti-Raymond M, David VA, Pflueger SM, Lindblad-Toh K, Wade CM, O'Brien SJ, Johnson WE. Patterns of molecular genetic variation among cat breeds. Genomics. 2008; 91(1): 1-11.

- Menotti-Raymond M, David VA, Schäffer AA, Tomlin JF, Eizirik E, Phillip C, et al. An autosomal genetic linkage map of the domestic cat, Felis silvestris catus. Genomics. 2009a; 93(4): 305-13.

- Menotti-Raymond M, David VA, Eizirik E, Roelke ME, Ghaffari H, O'Brien SJ. Mapping of the domestic cat "SILVER" coat color locus identifies a unique genomic location for silver in mammals. J Hered. 2009b; 100 Suppl 1(Suppl 1): S8-13. 
- Mériot M, Hitte C, Rimbault M, Dufaure de Citres C, Gache V, Abitbol M. Donskoy cats as a new model of oculocutaneous albinism with the identification of a splice-site variant in Hermansky-Pudlak Syndrome 5 gene. Pigment Cell Melanoma Res. 2020; 33(6): 814-825.

- Moses L, Niemi S, Karlsson E. Pet genomics medicine runs wild. Nature. 2018; 559(7715): 470-472.

- Mullikin JC, Hansen NF, Shen L, Ebling H, Donahue WF, Tao W, et al. Light whole genome sequence for SNP discovery across domestic cat breeds. BMC Genomics. 2010; 11: 406.

- Murphy WJ, Menotti-Raymond M, Lyons LA, Thompson MA, O'Brien SJ. Development of a feline whole genome radiation hybrid panel and comparative mapping of human chromosome 12 and 22 loci. Genomics. 1999; 57(1): 1-8.

- Murphy WJ, Sun S, Chen Z, Yuhki N, Hirschmann D, Menotti-Raymond M, O'Brien SJ. A radiation hybrid map of the cat genome: implications for comparative mapping. Genome Res. 2000; 10(5): 691-702.

- Murphy WJ, Davis B, David VA, Agarwala R, Schäffer AA, Pearks Wilkerson AJ, et al. A 1.5-Mb-resolution radiation hybrid map of the cat genome and comparative analysis with the canine and human genomes. Genomics. 2007; 89(2): 189-96.

- Nghiem PP, Kornegay JN. Gene therapies in canine models for Duchenne muscular dystrophy. Hum Genet. 2019; 138(5): 483-489.

- O’Brien SJ \& Nash WG. Genetic mapping in mammals: chromosome map of domestic cat. Science 1982; 216: 257-265.

- O’Brien SJ, Haskins ME, Winkler CA, Petterson DF. Chromosomal mapping of beta-globin and albino loci in the domestic cat. A conserved mammalian chromosome group. J Hered. 1986; 77: 374-378.

- O'Brien SJ, Johnson W, Driscoll C, Pontius J, Pecon-Slattery J, Menotti-Raymond M. State of cat genomics. Trends Genet. 2008; 24(6): 268-79.

- Oliveira R, Randi E, Mattucci F, Kurushima JD, Lyons LA, Alves PC. Toward a genome-wide approach for detecting hybrids: informative SNPs to detect introgression between domestic cats and European wildcats (Felis silvestris). Heredity (Edinb). 2015; 115(3): 195-205.

- Page JE \& Murphy WJ. Construction of radiation hybrid panels. Methods Mol Biol. 2008; 422: 51-64.

- Peterschmitt M, Grain F, Arnaud B, Deléage $G$, Lambert V. Mutation in the melanocortin 1 receptor is associated with amber colour in the Norwegian Forest Cat. Anim Genet. 2009; 40(4): 547-52.

- Pontius JU, Mullikin JC, Smith DR; Agencourt Sequencing Team, Lindblad-Toh K, Gnerre S, et al. Initial sequence and comparative analysis of the cat genome. Genome Res. 2007; 17(11): 1675-89.

- Pontius JU, O'Brien SJ. Artifacts of the $1.9 \mathrm{x}$ feline genome assembly derived from the feline-specific satellite sequence. J Hered. 2009; 100 Suppl 1(Suppl 1): S14-8.

- Prouteau A \& André C. Canine Melanomas as Models for Human Melanomas: Clinical, Histological, and Genetic Comparison. Genes (Basel). 2019; 10(7): 501.

- Steyer K, Tiesmeyer A, Muñoz-Fuentes $\mathrm{V}$, Nowak C. Low rates of hybridization between European wildcats and domestic cats in a human-dominated landscape. Ecol Evol. 2018; 8(4): 2290-2304.

- Story BD, Miller ME, Bradbury AM, Million ED, Duan D, Taghian T, et al. Canine Models of Inherited Musculos- keletal and Neurodegenerative Diseases. Front Vet Sci. 2020; 7: 80.

- Sun S, Murphy WJ, Menotti-Raymond $\mathrm{M}, \mathrm{O}$ 'Brien SJ. Integration of the feline radiation hybrid and linkage maps. Mamm Genome. 2001; 12(6): 436-41.

- Tamazian G, Simonov S, Dobrynin P, Makunin A, Logachev A, Komissarov A, et al. Annotated features of domestic cat - Felis catus genome. Gigascience. 2014; 3: 13.

- Tarone L, Barutello G, Iussich S, Giacobino D, Quaglino E, Buracco P, et al. Naturally occurring cancers in pet dogs as pre-clinical models for cancer immunotherapy. Cancer Immunol Immunother. 2019; 68(11): 1839-1853.

- Van der Weyden L, Brenn T, Patton EE, Wood GA, Adams DJ. Spontaneously occurring melanoma in animals and their relevance to human melanoma. J Pathol. 2020; 252(1): 4-21.

- Vigne JD, Guilaine J, Debue K, Haye L, Gérard P. Early taming of the cat in Cyprus. Science. 2004; 304(5668): 259. -Willmann M, Hadzijusufovic E, Hermine O, Dacasto M, Marconato L, Bauer K, et al. Comparative oncology: The paradigmatic example of canine and human mast cell neoplasms. Vet Comp Oncol. 2019; 17(1): 1-10.

- Wurster-Hill DH and Gray CW. Giemsa banding patterns in the chromosomes of twelve species of cats (Felidae). Cytogenet Cell Genet. 1973; 12: 388-397.

- Yu Y, Creighton EK, Buckley RM, Lyons LA; 99 Lives Consortium. A Deletion in GDF7 is Associated with a Heritable Forebrain Commissural Malformation Concurrent with Ventriculomegaly and Interhemispheric Cysts in Cats. Genes (Basel). 2020;11(6):672.

- Zhang W, Schoenebeck JJ. The ninth life of the cat reference genome, Felis_catus. PLoS Genet. 2020 Oct 22; 16(10): e1009045. 